\title{
Eqstudios EGerenciales
}

Journal of Management and Economics for Iberoamerica

Artigo de pesquisa

\section{Práticas para a gestão de custos logísticos em empresas industriais de grande porte da Colômbia}

Maryely Andrea Jimenez-Franco *

Pesquisadora, Programa de Pós-Graduação em Contabilidade, Universidade Federal de Santa Catarina, Santa Catarina, Brasil. pandorandrea@hotmail.com

Valdirene Gasparetto

Professora, Programa de Pós-Graduação em Contabilidade, Universidade Federal de Santa Catarina, Santa Catarina, Brasil. valdirene.gasparetto@ufsc.br

\section{Resumo}

Este estudo avalia a aplicação de práticas para gestão de custos logísticos (LCM) em empresas industriais de grande porte da Colômbia. Foi realizada survey, com 64 organizações, com análise quantitativa dos dados. Constatou-se que parte das organizaç̃es da amostra terceirizam as atividades logísticas e outras não possuem área correspondente formalmente constituída ou gestor responsável. As que possuem área de logística executam principalmente atividades mais tradicionais. A prática para LCM mais aplicada é Resposta Eficiente ao Consumidor, seguida de Custeio Baseado em Atividades. Esta pesquisa contribui com o avanço do conhecimento sobre LCM em organizações industriais, sobre práticas que contribuem para reduzir custos e melhorar o desempenho organizacional. Recomendam-se pesquisas futuras que analisem o fenômeno sob outras perspectivas considerando outros setores e práticas.

Palavras-chave: custos logísticos; gestão de custos logísticos; empresas industriais.

\section{Prácticas para la gestión de costos logísticos en grandes empresas industriales de Colombia}

\section{Resumen}

Éste estudio evaluó la aplicación de prácticas de gestión de costos logísticos en grandes empresas industriales de Colombia. Mediante un análisis cuantitativo, se aplicó una encuesta a 64 empresas. Se encontró que algunas tercerizan las actividades logísticas; otras no tienen un área formalmente constituida ni un responsable de tales actividades; y aquellas con área logística desarrollan las actividades más tradicionales. La práctica más aplicada es la respuesta eficiente al consumidor, seguida de los costos basados en las actividades. La presente investigación contribuye al avance del conocimiento sobre estas prácticas de gestión de costos logísticos en las organizaciones industriales y sobre las prácticas que contribuyen a reducir costos y a mejorar el desempeño organizacional. Se recomienda futuras investigaciones para analizar el fenómeno desde otras perspectivas considerando otros sectores y prácticas.

Palabras clave: costos logísticos; gestión de costos logísticos; empresas industriales.

\section{Practices for logistic costs management in large industrial companies in Colombia}

\section{Abstract}

This study assessed the application of practices for logistic costs management in large industrial companies in Colombia. Through a quantitative analysis, a survey was carried out with 64 companies. It was found that some of these companies outsource logistic activities; others do not have a formally constituted area or a responsible manager for such activities, and those with a logistic area perform mainly more traditional activities. The most applied practice is efficient consumer response, followed by activity-based costing. This research contributes to the advancement of knowledge about these logistic costs management practices in industrial organizations and about practices that contribute to reduce costs and improve organizational performance. Future research is recommended to analyze the phenomenon from other perspectives considering other sectors and practices.

Keywords: logistic costs; logistic costs management; industrial companies.

* Autor para dirigir correspondência

Classificações JEL: L60; M40; Y40.

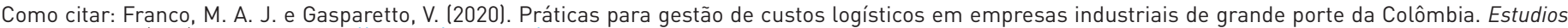
Gerenciales, 36(156), 364-373. https://doi.org/10.18046/j.estger.2020.156.3754

DOI: https://doi.org/10.18046/j.estger.2020.156.3754

Recebido: 28-out-2019

Aceito: 21 -jul-2020

Publicado: 28 -set-2020 


\section{Introdução}

No cenário competitivo recente, as organizações enfrentam crescente concorrência econômica, pressão para reduzir custos, exigências dos clientes por produtos que atendam às suas necessidades em termos de qualidade, funcionalidade e preço, e pressão dos acionistas por rentabilidade que reflita seu risco (Gonçalves, Gaio \& Silva, 2018).

Nesse contexto, informações para gestão oriundas de métodos tradicionalmente utilizados pela contabilidade das organizações podem não ser suficientes para atender as diversas demandas, inclusive as que se referem a custos de processos, de produtos, de fornecedores e de compradores, e também os custos relativos à logística das organizações (Castro, Camelo \& Ospina, 2016), foco deste estudo.

Em relatórios contábeis padronizados, a visibilidade dos custos logísticos é baixa, pois estão dispersos em todas as categorias de custos e sem rubricas específicas, o que dificulta sua adequada identificação e análise (Dianwei, 2006; Slusarczyk, 2014).

A gestão de custos logísticos llogistics cost management ou LCM) busca melhorar a visibilidade de atividades e custos logísticos, e permite análises do resultado para o nível de clientes, segmentos, canais de distribuição, áreas de negócios e a organização como um todo (Krajnc, Logožar \& Korošec, 2012; Silva, Gonçalves \& Leite, 2014), a partir de técnicas para identificação e gestão de custos logísticos (Yang, 2011; Slusarczyk, 2014, Škerlič \& Muha, 2016).

Práticas contábeis e de gestão de custos podem contribuir para a LCM. Estudos anteriores propõem a aplicação do custeio baseado em atividades lactivity-based costing ou $A B C$ ) na LCM para melhorar a visibilidade e determinação dos custos das atividades logísticas e auxiliar na obtenção de informações mais adequadas e no controle desses custos IStapleton, Pati, Beach \& Julmanichoti, 2004; Dianwei, 2006; Xiao, Sun \& Shi, 2009; Krajnc et al., 2012). Outros estudos propõem custeio baseado em atividade e tempo (time-driven activity based costing ou TDABC), rentabilidade direta do produto (direct product profitability ou DPP), custos para servir (cost to serve ou CTS) e resposta eficiente ao consumidor (efficient consumer response ou ECR), como em Guerreiro, Rodrigues e Vazquez (2008), Yang (2011), Souza, Zwirtes, Rodniski e Borghetti (2013a), Souza, Schnorr e Ferreira (2013b), Silva et al. (2014), Souza, Rempel e Silva (2014), Souza, Weber e Campos (2015), Campelo e Faustino (2015), Škerlič e Muha (2016) e Vargas, Coser e Souza (2016). Uyar (2014) propõe a aplicação do custo total de propriedade (total cost of ownership ou TCO). Além desses, Márquez, Pardo e Nieto (2015) mostram a análise da rentabilidade do cliente (consumer profitability analysis ou CPA) para reduzir custos logísticos e aumentar os lucros, ao permitir a tomada de decisões sobre clientes não rentáveis.

Ao analisar o âmbito empresarial da indústria da Colômbia, identificou-se poucas pesquisas acerca de custos logísticos. Jaimes, Díaz e Serna (2011), González (2014), Ibargüen e Cortés (2012) e Argueta, Cardona, Albán e Moreno (2015) apresentam estudos de casos, relatos de experiências e proposições de modelos para reduzir custos da logística, porém não se identificou estudos que abordaram LCM. Assim, a pesquisa apresentada neste artigo contribui para esse fluxo de estudos no País, a partir do objetivo de identificar a aplicação de práticas para gestão de custos logísticos (LCM) em organizações industriais de grande porte da Colômbia. Para alcançar o objetivo, foram analisadas as características da distribuição, grau de assimetria dos dados e feita a correlação de Spearman, para verificar a relação entre a aplicação das práticas a partir da terminologia pela qual são conhecidas na literatura da área, e a efetiva implementação dessas práticas a partir das suas características e funcionalidades, na gestão dos custos logísticos das empresas da amostra.

0 restante deste artigo está organizado da seguinte forma: na seção dois, se apresenta o referencial teórico; na seção três, os procedimentos metodológicos; na seção quatro, os resultados e discussão; e na seção cinco, as conclusões do estudo.

\section{Referencial teórico}

\subsection{Atividades e custos logísticos}

A percepção de cada organização sobre o conceito de logística influencia na definição de quais atividades são consideradas como parte da logística, e também se o custo dessas atividades será monitorado e/ou explicitamente reconhecido como um custo logístico.

Durante a década de 1950 as organizações gerenciavam as atividades logísticas sob a responsabilidade de diferentes áreas: (i) transporte estava sob o comando da gestão da produção; (ii) gestão de estoques era responsabilidade de marketing, finanças ou produção; e (iii) processamento de pedidos pertencia a finanças ou produção, gerando conflitos de objetivos e responsabilidades para essas atividades de logística (Ballou, 1993). Ao longo dos anos 1960, essas atividades passaram a ser identificadas e atribuídas à logística, permitindo incluir as necessidades dos clientes como foco dessas atividades (Servera-Francés, 2010; Islam, Meier, Aditjandra, Zunder \& Pace, 2013).

Mais recentemente, as atividades logísticas passaram a ser classificadas segundo sua natureza e custos relacionados em: (i) primárias ou comuns e (ii) secundárias, de apoio ou especiais. Primárias são as atividades essenciais à coordenação e cumprimento da tarefa logística, respondendo pela maior parte do seu custo total (Ballou, 1993; Škrinjar, Ivaković \& Šimunović, 2008; Servera-Francés, 2010), envolvendo custos de transporte, gestão de estoques e processamento de pedidos. Atividades secundárias são atividades adicionais que podem gerar custos logísticos (Ballou, 1993; Škrinjar et al., 2008), com serviço ao cliente, armazenagem, gestão de materiais, obtenção ou aquisição, planejamento da produção, gestão da informação, logística reversa, entre outras.

Dentre as atividades logísticas primárias, transporte (i) é a mais importante em algumas organizações, por ser responsável pela movimentação de matérias-primas e produtos acabados por diversos modais, a partir da infraestrutura de transporte, considerando condição 
geográfica, tipo de entrega, planejamento, roteamento e agendamento (Islam et al., 2013), e pode gerar custos logísticos elevados; gestão de estoques (ii) envolve a gestão de matérias-primas e produtos finais, previsão de vendas, lista de produtos em armazéns, número, tamanho e localização dos pontos de armazenamento e estratégias para receber e enviar produtos de armazéns (Servera-Francés, 2010); enquanto processamento de pedidos (iii) envolve geração, preenchimento e entrega de pedidos aos clientes, devendo ser projetada uma rede e um processo que permita que a organização atenda todos os pedidos dos clientes, minimizando o custo total de entrega (Faria \& Costa, 2007; Škrinjar et al., 2008; Servera-Francés, 2010).

Dentre as atividades logísticas secundárias, as principais são: serviço ao cliente (i), que envolve custos de dispor o produto, confiabilidade do serviço em termos de qualidade e desempenho de tempo das entregas (Faria \& Costa, 2007); armazenagem (ii), que compreende administração do espaço necessário para manter estoque e demais custos envolvidos (Ballou, 1993; Servera-Francés, 2010); gerenciamento de materiais (iii), que é importante na seleção dos equipamentos de movimentação, procedimentos para requisição de materiais e balanceamento da carga de trabalho (Islam et al., 2013); e obtenção ou aquisição (iv), que compreende a seleção de fornecedores, quantidades a serem adquiridas, programação das compras e forma pela qual os materiais são obtidos (Servera-Francés, 2010).

Outras atividades logísticas secundárias incluem: planejamento da produção (v), que se refere às quantidades agregadas que devem ser produzidas, e quando e onde devem ser fabricados os produtos (Servera-Francés, 2010); gestão da informação (vi), que inclui processamento e manutenção da informação (Islam et al., 2013); logística reversa (vii), que se refere à gestão do "fluxo de matérias-primas, produtos e informações do ponto de consumo até o ponto de origem com a finalidade de recuperá-los ou dar-lhes um descarte adequado" (Faria \& Costa, 2007, p.27); e outras atividades (viii), que envolvem demais operações que ocorrem no macroprocesso logístico, como embalagem, unitização, coordenação com outros departamentos, coordenação com membros da cadeia, entre outras.

Em síntese, na logística empresarial há diversas atividades e custos que, para serem monitorados, requerem disposição de cada organização na sua identificação. 0 montante desses custos e percentual em relação aos custos totais depende de fatores como porte da organização, indústria de atuação e setor de atividade (Slusarczyk, 2014). Segundo Hälinen (2015), uma organização com práticas mais sofisticadas de gestão de custos logísticos tende a ter uma percepção mais ampla das atividades e dos custos envolvi-dos do que uma empresa com uma percepção de logística mais básica, podendo também ter uma gestão mais efetiva dos montantes envolvidos.

\subsection{Práticas para gestão de custos logísticos}

Gestão de custos logísticos llogistics cost management ou LCM) é um conceito complexo e abrangente (Song e Wang, 2009) e pode ser apoiado por práticas linstrumentos, artefatos, técnicas ou ferramentas) como ABC, TDABC, CPA, DPP, TCO, CTS e ECR (LaLonde \& Pohlen, 1996; Faria \& Costa, 2007; Krajnc et al., 2012; Souza et al., 2014; Campelo \& Faustino, 2015).

O ABC lactivity-based costing), ou custeio baseado em atividades, é um método de custeio que melhora a visibilidade dos custos logísticos em comparação aos métodos baseados em volume de produção, como o custeio por absorção, e permite avaliar os resultados da logística (Pohlen \& Lalonde, 1994; Faria \& Costa, 2007; Kang, 2015). Também leva a melhor compreensão dos custos da logística com base nas atividades (Stapleton et al., 2004; Dianwei, 2006) para melhorar a cadeia, reduzir o consumo de recursos, aumentar a qualidade operacional e gerenciar estrategicamente os custos ao longo de todo o ciclo de vida (Xiao et al., 2009, Krajnc et al., 2012).

Krajnc et al. (2012), em estudo numa fábrica de papel eslovena, abordam a implementação do custeio baseado em atividades ( $A B C$ ) na logística. Afirmam que o $A B C$ pode revelar muito mais custos indiretos respeito de um grupo de produtos do que o custeio tradicional (baseado em volume), garantindo informações mais completas de produtos em forma individual, o que permite o cálculo da rentabilidade por produto (Krajnc et al., 2012). Além disso, acreditam que a aplicação do ABC é mais eficiente e mais bem-sucedida para a gestão dos custos logísticos e permite: (i) utilizálo como ferramenta de suporte à tomada de decisões em relação a grupos de clientes ou clientes individuais; e (ii) permite medir e avaliar os efeitos das atividades logísticas.

Faria e Costa (2007), abordando vantagens do ABC na logística, citam a oportunidade de analisar a logística a partir da cadeia de suprimentos e a eliminação de atividades que não agregam valor. Como desvantagem, o ABC apresenta complexidade de implementação e análise por requerer muitos dados, levantamentos e controles. Öker e Adigüzel (2016) complementam afirmando que apesar dos benefícios do método, este não tem aceitação generalizada pois normalmente requer exames demorados e altos custos de processamento de dados.

Como uma variação do ABC, o TDABC Itime-driven activity based costingl é um método baseado em atividades, e orientado pelo tempo, que permite rastrear os custos logísticos a partir de estimativas de tempo de execução das atividades (Everaert, Bruggeman, Sarens, Anderson \& Levant, 2008; Namazi, 2016), e pode simplificar a alocação dos custos, reduzindo tempo e esforço exigidos pelo $A B C$.

Öker e Adigüzel (2016) afirmam que o TDABC elimina as dificuldades do ABC e atualiza o sistema de acordo com as mudanças das atividades com base em equações de tempo. Diferente do $\mathrm{ABC}$, no primeiro estágio de alocação, o TDABC atribui os custos indiretos não a atividades, mas para os departamentos, simplificando o processo e reduzindo tempo e esforço. Outro benefício é que permite a análise da utilização da capacidade, evidenciando a parte ociosa e, assim, contribui para eliminar o excesso de capacidade que gera custo extra para a organização lÖker \& Adigüzel, 2016). Porém, é preciso que a capacidade seja passível de mensuração pelo tempo, o que pode ser difícil em algumas organizações (Öker \& Adigüzel, 2016). 
O TCO (total cost of ownership), ou custo total de propriedade, identifica os custos referentes à aquisição de bens ou serviços de determinado fornecedor, envolvendo elementos anteriores à transação, durante e posteriores à transação, quando os itens já chegaram na empresa (Faria \& Costa, 2007; Campelo \& Faustino, 2015), e pode fornecer dados importantes para analisar, negociar e reduzir o custo total de compra e aumentar a rentabilidade (Uyar, 2014). Como benefício do TCO está a identificação de alternativas que levam à redução dos custos totais e ao provimento de informações úteis nas decisões de compra e de avaliação do desempenho de fornecedores (Faria \& Costa, 2007).

Ferrin e Plank (2002), usando TCO, avaliaram propostas de compras e observaram que um dos principais direcionadores desses custos é a logística, apesar disso, a contribuição dos gestores da logística para a avaliação de TCO é baixa, indicando ausência ou desconhecimento da ferramenta por parte desses executivos.

O CTS (cost-to-serve) destina-se a determinar os custos para servir os clientes. São identificadas as exigências para o atendimento de todos os clientes e o esforço necessário para atendê-los, levando em conta a quantidade de recursos humanos requeridos e demais custos envolvidos na transação (Guerreiro et al., 2008). Com essa informação, é possível operacionalizar a CPA (customer profitability analysis) ou análise da rentabilidade do cliente, uma prática operacionalizada a partir do cálculo da margem de contribuição ou da margem bruta dos produtos vendidos a um cliente, deduzidos os custos para servi-lo (Guerreiro et al., 2008).

Márquez et al. (2015), abordando a CPA em uma indústria alimentícia da Espanha, mostram que o instrumento permite mudar a forma como as organizações pensam sobre suas atividades logísticas. Com os dados da CPA de clientes, melhorou o roteamento de veículos e foram observados a redução nos custos logísticos em mais de $60 \%$ e o aumento nos lucros em mais de $75 \%$. A eliminação de clientes não rentáveis, utilizando a CPA, pode ter efeitos limitados nas vendas, mas custos comerciais e de transporte podem ser reduzidos.

Guerreiro et al. (2008), em empresa alimentícia do Brasil, empregaram o $\mathrm{ABC}$ no cálculo do CTS, e daí apuraram a CPA. Evidenciaram que a mensuração do CTS fornece informações específicas e detalhadas sobre o cliente, permitindo uma análise de rentabilidade do cliente mais abrangente do que o paradigma clássico (Guerreiro et al., 2008). Assim, tanto o ABC como o TDABC podem auxiliar na implementação de CTS e CPA.

A DPP (direct product profitability), ou rentabilidade direta do produto, identifica os gastos incorridos por produto nos diversos canais de distribuição, regiões ou clientes (Souza et al., 2013b). A DPP subtrai da margem de contribuição os custos diretamente atribuídos aos produtos, mostrando o lucro direto do produto (Silva et al., 2014). Song e Wang (2009), porém, lembram que os custos diretos da logística podem ser subtraídos do lucro de vendas com pouca consideração de custos indiretos, como monitoramento, administração, manutenção, compras e custo de manutenção de estoque, 0 que pode gerar informações frágeis para tomada de decisão.

ECR (efficient consumer response), ou resposta eficiente ao consumidor, é a técnica de integrar os elos da cadeia de suprimentos pela automação de atividades, eliminando transações manuais, que promovam o reabastecimento contínuo e a realização de ações conjuntas de vendas e cross-docking (LaLonde \& Pohlen, 1996), com o objetivo de melhorar a resposta aos clientes finais e reduzir custos logísticos (Ferreira \& Schnorr, 2011).

Para verificar nível de adoção de práticas de LCM, Souza et al. (2013a) realizaram um estudo de caso numa cooperativa agroindustrial e constataram a utilização de conceitos de TCO e ECR, mas de forma parcial, e o uso do custeio por absorção como método de apuração de custos, o que faz com que nem todos os gastos sejam considerados nos custos logísticos. Souza et al. (2013b), em outro estudo, analisaram uma empresa de alimentos e constataram que esta não utiliza o $A B C$, mas utiliza parcialmente $D P P$, CPA, TCO e ECR. Souza et al. (2014), em uma empresa de alimentos e bebidas, identificaram que o $A B C$ não é utilizado, e DPP e CPA são usados mas com potencial de melhoria, e TCO e ECR são utilizadas parcialmente. Souza et al. (2015), em uma empresa moveleira, identificaram a implantação de ERP e robotização dos processos de produção para buscar redução de custos, emprego parcial de TCO e custeio por absorção para apuração de custos, e Vargas et al. (2016), em uma indústria gráfica, verificaram que a empresa não adotava o $A B C$ impossibilitando a rastreabilidade dos custos, sendo utilizados parcialmente CPA, DPP, TCO, ECR e EDI.

As práticas descritas nesta seção possuem relação entre si, e sua combinação e integração como parte dos processos logísticos permite a compreensão do negócio, dos processos, canais e segmentos que têm importância estratégica e necessitam de reconfigurações e ajustes em termos de custos logísticos (Faria \& Costa, 2007; Fellous, 2009). Não há uma prática única que contemple o que se necessita para a tomada de decisões, assim, a incorporação de forma integrada e complementar das práticas para o gerenciamento dos custos logísticos poderia fornecer melhor suporte às organizações (Fellous, 2009; Silva et al., 2014).

\section{Procedimentos metodológicos}

Esta pesquisa é descritiva, com uma abordagem quantitativa do problema, que permite a análise das características gerais de empresas industriais de grande porte da Colômbia sobre a aplicação de práticas de gestão de custos logísticos a partir da coleta de dados (survey) realizada a partir da estrutura do instrumento de pesquisa que consta na tabela 1.

0 pré-teste do instrumento de pesquisa foi aplicado a um professor especialista da área de logística, gestão de operações e produção da Universidade Nacional da Colômbia, para identificar, corrigir e eliminar problemas potenciais de compreensão do instrumento, envolvendo o idioma espanhol, a formulação das questões e seu entendimento. Após 
Tabela 1. Instrumento de pesquisa

\begin{tabular}{|c|c|c|}
\hline Blocos & $\mathrm{N}^{0}$ das questões & Referências \\
\hline $\begin{array}{l}\text { Bloco I - Atividades que fazem parte da logística, área de } \\
\text { logística, número e qualificação dos colaboradores e foco } \\
\text { da LCM }\end{array}$ & $1,2 a-b-c, 3,4$ & $\begin{array}{c}\text { Fellous (2009); Amaral (2012); Servera-Francés (2010); } \\
\text { Islam et al. (2013); Nge, Rattanawiboonsom, Mahmood e } \\
\text { Rurkwararuk (2016). }\end{array}$ \\
\hline $\begin{array}{l}\text { Bloco II - Perguntas diretas sobre aplicação de práticas } \\
\text { para LCM, segundo nomenclatura usual na literatura - } \\
\text { ABC, TDABC, CTS, CPA, DPP, TCO e ECR. }\end{array}$ & 5 & $\begin{array}{l}\text { LaLonde e Pohlen (1996); Faria e Costa (2007); Everaert et } \\
\text { al. (2008); Guerreiro et al. (2008); Ferreira e Schnorr (2011); } \\
\text { Souza et al. (2014). }\end{array}$ \\
\hline $\begin{array}{l}\text { Bloco III - Perguntas indiretas sobre a aplicação de } \\
\text { práticas para LCM, de modo a verificar aplicação das } \\
\text { práticas com nomenclatura diversa daquela usual na } \\
\text { literatura. }\end{array}$ & $6,7 a-b, 8,9 a-b, 10,11,12 a-b-c$ & $\begin{array}{l}\text { Ferrin e Plank (2002); Xiao et al. (2009); Santos; Gonçalves, } \\
\text { Leite (2016); Souza et al. (2013b); Coser e Souza (2017). }\end{array}$ \\
\hline $\begin{array}{l}\text { Bloco IV - Perfil do respondente - sexo, idade, nível e } \\
\text { área de formação, cargo, e tempo de atuação. }\end{array}$ & $13,14,15,16,17,18,19$ & Elaboração própria \\
\hline Bloco V - Perfil da empresa. & $20,21,22$ & Elaboração própria \\
\hline
\end{tabular}

Fonte: elaboração própria.

realizar as adequações sugeridas, foi aplicado pré-teste em duas empresas da Colômbia, no período de 17 a 21 de julho de 2017, e identificou-se que o questionário abordava os termos de forma clara, não sendo necessárias outras alterações.

Para a seleção da população e amostra foi realizado um processo de filtragem e classificação em duas etapas. $\mathrm{Na}$ etapa 1, foram selecionadas, a partir da Lei 590 de 2000 (Congreso de Colombia, 2000), como população, 435 empresas industriais de grande porte localizadas principalmente nas cidades de Medellín, Cali, Bucaramanga e Bogotá Distrito Capital. Esta seleção justifica-se considerando que o custo que envolve a utilização de práticas para LCM ainda é considerado uma alternativa mais acessível às empresas de grande porte (Mejía-Argueta, Higuita-Salazar \& Hidalgo-Carvajal, 2015). Na etapa 2, fez-se contato com as empresas, por e-mail, obtendo resposta de 176 empresas, às quais foi solicitado acesso ao profissional de logística, ou gestor encarregado de administrar essa área, para convidálo a participar da pesquisa, e 64 empresas aceitaram participar da pesquisa, compreendendo a amostra do estudo.

Para a coleta dos dados, foi enviado aos participantes o instrumento por meio do Software SurveyMonkey ${ }^{\circledR}$, disponível no período do 26 de novembro de 2017 a 15 de janeiro de 2018.

As questões dos blocos I, IV e $\mathrm{V}$ do instrumento de pesquisa foram analisadas a partir de análise descritiva e quantitativa. As questões do bloco II foram analisadas pelas características da distribuição e grau de assimetria dos dados, pois nem todas as empresas aplicam as práticas de LCM com o mesmo grau de intensidade, fato que implicou em estabelecer uma escala quantitativa para determinar o grau de aplicação das práticas nas empresas respondentes. As questões do bloco III foram analisadas pela correlação de Spearman, para verificar a relação entre a aplicação das práticas da forma como o termo é conhecido na literatura (perguntas diretas) e a aplicação efetiva (perguntas indiretas) de práticas a partir de suas características, na gestão de custos logísticos.

\section{Resultados e discussão}

\subsection{Perfil dos respondentes e das empresas}

Das 64 empresas que participaram da pesquisa, $18(28,1$ $\%)$ afirmaram ter logística terceirizada e $9(14,1 \%)$ afirmaram não possuir área de logística formalmente constituída ou gestor responsável, e por isso não responderam a totalidade do instrumento. Esses achados vão de encontro à literatura da área, que afirma que cerca de $40 \%$ da logística global é terceirizada (Wong, Maher, Nicholson \& Gurney, 2000).

A decisão pela terceirização logística pode ser decorrente de análise de custo-benefício, pois a terceirização permite liberação do capital interno para dedicá-lo ao negócio principal, e manter uma área funcional para a logística implica aumento de custos fixos e a necessidade de gerenciar mais ativos (González, 2015) e, nessas decisões, as empresas avaliam a "experiência e especialização do operador logístico, que eleva a qualidade, reduz a necessidade de investimentos em ativos, e facilita a gestão de custos" (Fellous, 2009, p. 171).

Wong et al. (2000) afirmam que uma organização pode buscar terceirização da logística por um ou mais motivos: sua falta de expertise em logística, desejo de concentrar-se em suas competências principais, dificuldade em manter comunicação e tecnologia da informação adequadas, interesse em melhorar as capacidades da cadeia de suprimentos como um todo, e a busca de flexibilidade.

Também é possível que empresas da amostra realizem internamente algumas atividades mais tradicionais de logística, como transporte, armazenagem, abastecimento e gestão de estoques, mas não as considerem como parte do processo da logística, por falta de compreensão do conceito de logística ou pela não existência de uma área com tal denominação, já que as atividades de logística podem estar sob a coordenação de outras áreas da organização.

As organizações que afirmaram ter uma área de logística e gestor responsável somaram 37 157,8\% da amostra), predominando no perfil dos gestores o gênero 
masculino $(64,9 \%)$. Este achado denota que ainda existe uma forte liderança no setor da logística de homens, achados congruentes com o estudo de Nigam (2010), que entrevistou 70 mulheres do setor de logística em Nova Deli, na Índia, e observou que apesar da presença de mulheres no mundo corporativo, na logística e transporte o número ainda é muito baixo, assim como são poucas as que ocupam cargos de gestão ou de alto escalão (Nigam, 2010).

Os respondentes possuem predominantemente ensino superior, com formação em administração; com faixa etária predominante de 30 a 39 anos; $27,0 \%$ são coordenadores logísticos, $16,2 \%$ são diretores e 10,8\% são chefes de logística; e $67,5 \%$ dos 37 respondentes possuem experiência na área de 3 a 10 anos. 0 setor econômico predominante é o de bens de consumo duráveis $(59,5 \%)$ e predominam empresas com sede em Medellín $170,3 \%$ da amostral onde, conforme a Dirección de Impuestos y Aduanas Nacionales de Colombia (DIAN, 2016), se encontra a maior parte das empresas do setor industrial da Colômbia. Dos respondentes, $48,6 \%$ atuam em mercados internacionais.

\subsection{Atividades logísticas e foco da gestão de custos logísticos}

Segundo Servera-Francés (2010) e Islam et al. (2013), nenhuma operação logística poderia transcorrer eficientemente sem gestão de estoques, armazenagem e gestão da informação. Dos respondentes, 97,3\% citam essas atividades como parte da logística; $86,5 \%$ citam transporte e processamento de pedidos; $83,8 \%$ citam outras atividades como embalagem e coordenação com outros departamentos; e $73,0 \%$ mencionam coordenação com outros membros da cadeia. Serviço ao cliente, apesar de considerado o articulador das atividades logísticas (Islam et al., 2013), foi mencionado por $70,3 \%$ dos respondentes, mesma proporção do gerenciamento de materiais. Contrário às tendências atuais e às políticas de proteção do meio ambiente que aumentam a sustentabilidade e consciência ecológica empresarial, a logística reversa foi citada por $59,5 \%$ dos respondentes, sendo uma das atividades menos consideradas como parte da logística, mesmo percentual de planejamento da produção.

Neste estudo, 59,5\% dos respondentes afirmaram ter área de logística formalmente estabelecida, em contraste aos achados de Fellous (2009) e Amaral (2012), onde 86,0\% e $98,6 \%$ das empresas, respectivamente, possuíam essa área; quanto ao número de colaboradores na área, $72,7 \%$ dos respondentes possuem entre 20 e 100 colaboradores; com qualificação considerada pelos respondentes como aceitável para desenvolver-se no setor $(5,9$ numa escala de 0 a 10 , com desvio padrão de 1,3). Esse nível de qualificação pode repercutir no desempenho da logística, pois conforme $\mathrm{Nge}$ et al. (2016), a capacitação, o desenvolvimento de habilidades, as competências na fabricação ou prestação do serviço, e a experiência e conhecimento da cadeia de suprimentos podem afetar as atividades logísticas.

A pesquisa também identificou o foco para gestão dos custos logísticos nas organizações pesquisadas. Dentre das alternativas fornecidas, numa escala de 0 a 10 , foram mais citadas pelos respondentes: melhoria do resultado da empresa $(7,0)$; racionalizar os custos de produção e reduzir custos logísticos (6,9); otimizar o custo total dos processos logísticos (6,7); evitar rupturas $(6,0)$; reduzir investimentos em ativos logísticos $(5,3)$; e reduzir os níveis de inventário $(5,2)$

\subsection{Aplicação de práticas de LCM, identificadas segundo nomenclatura usual na literatura}

Foi solicitado aos respondentes que identificassem a aplicação das práticas para LCM, perguntando de forma direta sobre a prática com a nomenclatura pela qual é usualmente denominada na literatura. Os resultados constam na tabela 2.

Conforme tabela 2, ECR apresenta a prática com maior grau de aplicação pelas empresas da amostra $(6,4)$, apesar de dispersão considerável nas respostas, com desvio padrão de 3,1, como se observa na figura 1.

A distribuição das respostas sobre ECR também apresenta uma assimetria considerável à esquerda $(-0,8)$, pois 24 respondentes afirmaram aplicar a prática ECR frequentemente ou sempre. A prática $A B C$, apesar de a literatura indicar sua contribuição na identificação dos custos logísticos, foi a segunda mais aplicada pelas empresas da amostra, como se observa na tabela 2, e, embora a média tenha sido 5,2 , a dispersão das respostas foi de 2,5 pontos. A prática menos utilizada foi o TDABC.

Tabela 2. Práticas aplicadas para LCM segundo a nomenclatura usual na literatura

\begin{tabular}{lccc}
\hline Prática & Média & Desvio padrão & Assimetria \\
\hline ECR & 6,4 & 3,1 & $-0,8$ \\
ABC & 5,2 & 2,5 & $-0,7$ \\
CTS & 5,1 & 3,5 & $-0,3$ \\
DPP & 4,5 & 3,8 & 0,2 \\
CPA & 4,0 & 3,6 & 0,3 \\
TCO & 2,8 & 3,0 & 0,9 \\
TDABC & 2,0 & 3,2 & 1,4 \\
\hline
\end{tabular}

Legenda: Escala de 0 a 10, onde 0 (não aplica), 1-2 (aplica muito pouco), 3-4-5 (aplica pouco), 6-7-8 (frequentemente), 9-10 (sempre). Utilização de práticas para LCM $(n=34)$.

Fonte: elaboração própria.

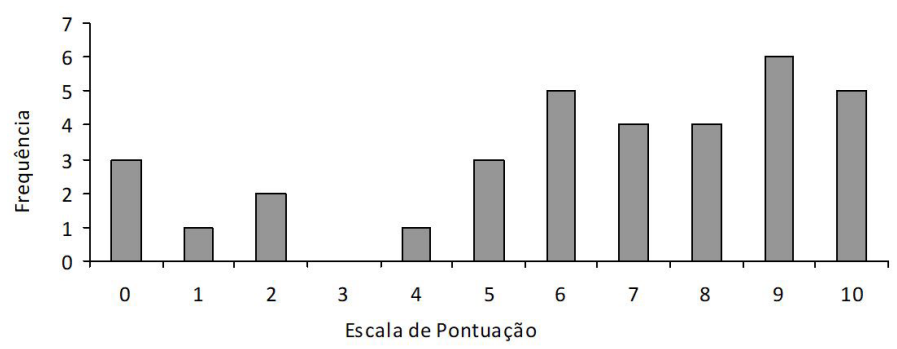

Figura 1. Distribuição das respostas acerca da aplicação da prática ECR Fonte: elaboração própria. 


\subsection{Aplicação de práticas de LCM, identificadas a partir de suas características}

Quando lhes perguntamos aos gestores sobre a aplicação de práticas para gestão de custos logísticos, a partir de perguntas indiretas sobre as características dessas práticas, sem que fosse mencionado o termo mais usual na literatura, os resultados constam na tabela 3 .

$\mathrm{Na}$ tabela 3 observa-se as perguntas indiretas sobre a aplicação das práticas, a partir de suas características. Novamente a ECR consta como a prática mais adotada nível 6,8 em escala de zero a 10. A questão 12a menciona que os respondentes automatizam as operações de logística, buscando a eliminação das transações manuais, como parte da resposta eficiente ao consumidor. Quando os resultados são comparados aos da pergunta direta, percebe-se que as respostas não foram influenciadas pelo entendimento do termo. Em contraste, o TDABC (questões 7a e 7b) foi a prática de menor pontuação $(3,4)$, também

Tabela 3. Práticas aplicadas para LCM a partir de perguntas indiretas

\begin{tabular}{lcccc}
\hline n(34) & $\begin{array}{c}\text { Prática relacionada à } \\
\text { questão }\end{array}$ & Média & $\begin{array}{c}\text { Desvio } \\
\text { padrão }\end{array}$ & Assimetria \\
\hline Questão 12a & ECR & 6,8 & 1,5 & $-1,5$ \\
Questão 12c & ECR & 6,5 & 1,6 & 0 \\
Questão 6 & ABC & 6,2 & 2 & 0 \\
Questão 12 b & ECR & 6,2 & 2,1 & $-0,6$ \\
Questão 9b & CTS & 5,9 & 2,3 & $-0,2$ \\
Questão 11 & DPP & 5,4 & 2,6 & 0,2 \\
Questão 10 & TCO & 5 & 2,8 & $-0,1$ \\
Questão 9a & CTS & 4,7 & 2,5 & 0,2 \\
Questão 8 & CPA & 4,6 & 2,5 & 0,5 \\
Questão 7a & TDABC & 3,4 & 3 & 0,5 \\
Questão 7b & TDABC & 3,4 & 3,3 & 0,6 \\
\hline
\end{tabular}

Legenda: Escala de 0 a 10, onde 0 (não aplica), 1-2 (aplica muito pouco), 3-4-5 (aplica pouco), 6-7-8 (frequentemente), 9-10 (sempre). Utilização de práticas para LCM (n=34).

Perguntas indiretas: Questão 6 (Monitora processos e atividades, apura custos e aloca com direcionadores de custos, para apurar custos logísticos de produtos); Questão 7a (Gerencia custos de atividades executadas estimando o tempo consumido na logística); Questão 7b (Utiliza informações de tempo das atividades da logística para obter informações para medição do desempenho logístico); Questão 8 (Associa custos logísticos a cada cliente, como parte da análise da lucratividade por cliente); Questão 9a (Possui informação de custos das atividades para servir cada cliente: custo do produto, despesas comerciais relacionadas com clientes e custo logístico); Questão 9b (Adota ações para reduzir custos das atividades de atendimento aos clientes); Questão 10 (Considera na aquisição de um item todos os custos associados à compra: de transação, de qualidade, do ciclo de vida do produto; de manutenção, reparo e operação; com gestão de pessoal; e não somente o preço de compra dos itens); Questão 11 (Identifica a margem de contribuição direta do produto, considerando receita e custos diretos incorridos para produção de um item específico); Questão 12a (Automatiza operações de logística, eliminando transações manuais, como parte da resposta eficiente ao consumidor). Questão 12 b (Integra fornecedores como parte da cadeia de suprimentos para tornar o reabastecimento contínuo, realizar ações conjuntas de compra e cross-docking); Questão 12 c (Integra clientes como membros da cadeia de suprimentos, para melhorar a resposta eficiente ao consumidor final).

Fonte: elaboração própria. alinhada à resposta da pergunta direta acerca da aplicação desta prática, o que confirma sua baixa aplicação nas empresas da amostra.

Em relação ao $A B C(6,2)$, a dispersão das respostas de 2,0 pontos e alta convergência ao valor médio mostrou que os respondentes se posicionaram de formas bastante diversas sobre esta prática, adotada pouco ou frequentemente.

Para verificar a relação e coerência nas respostas entre as práticas pesquisadas, considerando a nomenclatura da literatura (Bloco II) e as perguntas indiretas (Bloco III), foi realizada a matriz de correlação, conforme tabela 4 .

A tabela 4 mostra correlação moderada $(\rho=0,516)$, ao nível de significância de 1\%, entre o $\mathrm{ABC}$ e a questão indireta correspondente (Q.6), indicando coerência nas respostas. Em relação ao TDABC, observa-se correlação moderada $(\rho=0,643)^{\prime}$ com a questão $7 a$ e correlação forte $(\rho=0,730)$ com a questão 7b, ambas com nível de significância inferior a $1 \%$, ou seja, os respondentes que apontaram a aplicação desta prática a relacionam com a implementação nos custos das atividades logísticas, calculando o tempo consumido nessas atividades e obtendo informações para medição do desempenho logístico.

Apesar de não ter estabelecido um relacionamento, a priori, entre a pergunta direta do TDABC e a questão 9b (pergunta indireta do CTS), observou-se correlação moderada $(\rho=0,669)$, o que indica que os respondentes associaram a aplicação da prática TDABC à implementação de ações para reduzir os custos das atividades de atendimento aos clientes.

A CPA (pergunta direta) apresentou correlação moderada $(\rho=0,575)$ com a pergunta indireta correspondente 8 , que investigava se associavam os custos logísticos a cada cliente, como parte da análise da lucratividade por cliente. Com nível significância inferior a $1 \%$, os respondentes apontaram $\mathrm{v}$ ários graus de aplicação entre não aplicar, aplicar pouco ou muito pouco a prática CPA para a LCM, corroborando os resultados dos estudos de Souza et al. (2013b), Souza et al. (2014) e Vargas et al. (2016), nos quais a prática foi identificada como não utilizada, utilizada pouco ou parcialmente.

Por outro lado, foi identificada correlação forte $(\rho=0,756)$ entre a CPA e a questão 9a, sobre existência de informação de custos das atividades para servir cada cliente, como custo do produto, despesas comerciais relacionadas com clientes e custo logístico (pergunta indireta do CTS), ainda que não se tenha estabelecido relacionamento, a priori, entre ambas. Evidenciou-se que a CPA está fortemente relacionada à informação de custos para servir os clientes, o que pode decorrer de a CPA ser determinada a partir da margem bruta ou de contribuição dos produtos vendidos a um cliente, deduzidos os custos incorridos para servi-lo (Guerreiro et al., 2008).

O CTS (pergunta direta) apresentou correlação moderada com a questão 9b, que indagava sobre a adoção de ações para reduzir custos das atividades de atendimento aos clientes $(\rho=0,529)$, ao nível de significância de $1 \%$, e mostrou relação moderada e correlação fraca $(\rho=0,453)$, com nível significância inferior a 1\% na questão 9a. 
Tabela 4. Matriz de correlação

\begin{tabular}{|c|c|c|c|c|c|c|c|c|c|c|c|}
\hline Práticas $n=34$ & $\begin{array}{l}\text { Q.6 } \\
\text { ABC }\end{array}$ & $\begin{array}{l}\text { Q.7a } \\
\text { TDABC }\end{array}$ & $\begin{array}{l}\text { Q7b } \\
\text { TDABC }\end{array}$ & $\begin{array}{l}\text { Q.8 } \\
\text { CPA }\end{array}$ & $\begin{array}{l}\text { Q.9a } \\
\text { CTS }\end{array}$ & $\begin{array}{l}\text { Q.9b } \\
\text { CTS }\end{array}$ & $\begin{array}{l}\text { Q.10 } \\
\text { TCO }\end{array}$ & $\begin{array}{l}\text { Q.11 } \\
\text { DPP }\end{array}$ & $\begin{array}{l}\text { Q.12a } \\
\text { ECR }\end{array}$ & $\begin{array}{l}\text { Q.12b } \\
\text { ECR }\end{array}$ & $\begin{array}{l}\text { Q.12c } \\
\text { ECR }\end{array}$ \\
\hline \multicolumn{12}{|l|}{$A B C$} \\
\hline Nível Sig.1 & 0,001 & 0,403 & 0,340 & 0,072 & 0,017 & 0,073 & 0,019 & 0,407 & 0,091 & 0,108 & 0,044 \\
\hline \multicolumn{12}{|l|}{ TDABC } \\
\hline Nível Sig.1 & 0,001 & 0,000 & 0,000 & 0,011 & 0,083 & 0,000 & 0,003 & 0,000 & 0,003 & 0,000 & 0,004 \\
\hline \multicolumn{12}{|l|}{ CPA } \\
\hline Coeficiente Spearman & $0,433^{* *}$ & $0,500^{* *}$ & $0,303^{*}$ & $0,575^{* *}$ & $0,756^{* *}$ & $0,521^{* *}$ & $0,614^{* *}$ & $0,473^{* *}$ & 0,138 & $0,301 *$ & $0,344^{*}$ \\
\hline Nível Sig.1 & 0,005 & 0,001 & 0,041 & 0,000 & 0,000 & 0,001 & 0,000 & 0,002 & 0,219 & 0,042 & 0,023 \\
\hline \multicolumn{12}{|l|}{ CTS } \\
\hline Coeficiente Spearman & $0,412^{* *}$ & $0,315^{*}$ & 0,283 & $0,462^{* *}$ & $0,445^{* *}$ & $0,344^{*}$ & $0,381 *$ & 0,258 & 0,157 & $0,314^{*}$ & $0,323^{*}$ \\
\hline Nível Sig.1 & 0,008 & 0,035 & 0,052 & 0,003 & 0,004 & 0,023 & 0,013 & 0,070 & 0,187 & 0,035 & 0,031 \\
\hline \multicolumn{12}{|l|}{ DPP } \\
\hline Coeficiente Spearman & $0,506^{* *}$ & $0,529 * *$ & $0,529 * *$ & $0,469 * *$ & $0,413^{* *}$ & $0,619 * *$ & $0,377^{*}$ & $0,565^{* *}$ & $0,316^{*}$ & $0,496^{* *}$ & $0,396^{*}$ \\
\hline Nível Sig.1 & 0,001 & 0,001 & 0,001 & 0,003 & 0,008 & 0,000 & 0,014 & 0,000 & 0,034 & 0,001 & 0,010 \\
\hline \multicolumn{12}{|l|}{ ECR } \\
\hline Coeficiente Spearman & $0,522^{* *}$ & 0,005 & 0,036 & $0,456^{* *}$ & $0,458^{* *}$ & $0,392^{*}$ & $0,396 *$ & 0,103 & 0,203 & $0,489 * *$ & $0,537^{* *}$ \\
\hline Nível Sig.1 & 0,001 & 0,489 & 0,420 & 0,003 & 0,003 & 0,011 & 0,010 & 0,282 & 0,125 & 0,002 & 0,001 \\
\hline
\end{tabular}

Legenda: *Correlação significativa ao nível de $5 \% .{ }^{* *}$ Correlação significativa ao nível de $1 \%$. Os valores marcados associam-se a variáveis que, a priori, possuem correlação.

Fonte: elaboração própria.

A prática TCO (pergunta direta) apresentou correlação fraca ( $p=0,381)$, com nível de significância inferior a $5 \%$, com a pergunta indireta (questão 10) que perguntava se os respondentes consideram, na aquisição de um item, todos os custos associados à compra (transação, qualidade, ciclo de vida do produto, manutenção, reparo, operação, gestão de pessoal) e não somente o preço de compra dos itens, havendo grande discrepância nas respostas obtidas.

A DPP apresentou correlação moderada $(\rho=0,565)$, ao nível de significância de 1\%, com a questão 11 , havendo consenso parcial entre as respostas obtidas na pergunta direta e indireta. A prática apresentou correlação moderada ( $p=0,619)$, ao nível de significância de $1 \%$, com a questão $9 \mathrm{~b}$ (pergunta indireta do CTS), possivelmente por ser uma prática de gestão que identifica custos incorridos por produto nos diversos canais de distribuição, regiões ou clientes (Souza et al., 2013b), neste caso, os custos das atividades de atendimento a cada cliente.

A prática ECR apresentou correlação moderada $(\rho=0,537)$ com a questão 12c (pergunta indireta), com nível de significância de $1 \%$. Portanto, há correspondência entre os valores médios obtidos das pontuações dos respondentes para a aplicação (média=6,41) e a questão 12c (média=6,50) da ECR, mostrando que os respondentes apontaram a aplicação em um grau entre frequentemente ou sempre e associaram a aplicação da ECR à integração dos clientes como membros da cadeia de suprimentos, para melhorar a resposta eficiente ao consumidor, sendo essa, portanto, a prática mais implementada pelos respondentes.

\section{Conclusões}

Este estudo objetivou identificar a aplicação de práticas para gestão de custos logísticos (LCM) em organizações industriais de grande porte da Colômbia. Os resultados obtidos a partir da aplicação dos questionários mostraram que parte das empresas têm logística terceirizada, seguindo tendência mundial de transferir essas atividades a outras organizações (operadores logísticos), e outras afirmaram não possuir área de logística formalmente constituída ou gestor de logística.

Dentre as que possuem área de logística, predominam as que possuem entre 51 a 100 colaboradores na área, principalmente homens com faixa etária predominante entre 30 a 39 anos, com formação predominantemente em administração de empresas. A maior parte das empresas está localizada em Medellín, polo industrial da Colômbia.

Quanto às atividades logísticas, as empresas da amostra indicaram principalmente desenvolver gestão de estoques, armazenagem e gestão da informação, seguidas por transporte, processamento de pedidos, embalagem e empacotamente e coordenação com outros departamentos 
da empresas, todas com índices de adoção superiores a $80 \%$ pelas empresas da amostra. Porém, poucas indicaram desenvolver atividades de logística reversa, apesar da tendência atual no cuidado e conservação do meio ambiente. Constatou-se que os principais focos para a gestão dos custos logísticos nas organizações pesquisadas são melhoria do resultado, racionalização de custos de produção e redução de custos logísticos.

Os resultados mostraram que a prática mais adotada nas organizações da amostra é a resposta eficiente ao consumidor (ECR), o que mostra ênfase na automatização de processos, integração da cadeia de suprimentos e incorporação de práticas que permitam resposta rápida no fornecimento de produtos ou serviços aos consumidores. Em estudos desenvolvidos no Brasil (Souza et al., 2013a; Souza et al., 2013b; Souza et al., 2014; Vargas et al., 2016), a ECR também se apresentou como a prática mais presente nas organizações, porém, com aplicação ou uso parcial.

Verificou-se que o ABC, nas organizações da amostra, possui aplicação entre pouca e frequente, resultados coerentes com estudos prévios (Souza et al., 2013a; Souza et al., 2014; Souza et al., 2015; Campelo \& Faustino, 2015; Vargas et al., 2016), em que também constatou-se baixa adoção, apesar da literatura mencioná-lo como aceito pelos gestores da logística por permitir melhor alocação dos custos, melhorando a visibilidade e o controle dos custos na logística, conforme Stapleton et al. (2004), Xiao et al. (2009) e Krajnc et al. (2012). Diversas práticas de LCM discutidas neste estudo necessitam de informações de custos das atividades desempenhadas para sua operacionalização, e o $A B C$ seria um instrumento adequado para obter informações de custos que poderiam ser base para a utilização de outras práticas como CTS, DPP, CPA e, inclusive, para apurar custos para o TCO.

Por outro lado, constatou-se que a prática TDABC é pouco aplicada nas organizações da amostra, corroborando a constatação de Öker e Adigüzel (2016), de ser uma prática mais adequada e fácil de implementar por empresas de serviço do que empresas manufatureiras. Do mesmo modo, a CPA não é aplicada ou aplicada muito pouco nas organizações pesquisadas, o que difere de outros estudos como 0 de Fellous (2009), no qual a CPA foi a prática mais utilizada - por $74 \%$ da amostra. Os mesmos achados foram obtidos com as práticas CTS, DPP e TCO, que se apresentaram como não aplicados ou aplicados muito pouco pelos respondentes. Conclui-se, assim, que o grau de aplicação da maioria das práticas pesquisadas é entre frequente, pouco ou não aplicada, excetuando-se ECR e ABC, que apresentaram maior grau de adoção nas organizações pesquisadas.

Esta pesquisa contribui com o avanço do conhecimento sobre adoção de práticas de gestão de custos logísticos em organizações industriais, práticas essas capazes de apoiar as organizações na redução de custos e melhoria do seu desempenho. Mais pesquisas nesse tema são úteis para aprofundar a compreensão sobre a gestão de custos logísticos em organizações da Colômbia, e alavancar a gestão da logística no País.
Embora o estudo revele descobertas importantes sobre LCM em organizações colombianas, deve ser considerado seu escopo limitado, devido ao número de respondentes do setor industrial da Colômbia, dificultando a generalização dos achados. 0 questionário também limita os resultados, na medida em que poderia haver outras práticas implementadas e que não foram consideradas nesta pesquisa. Diante das limitações, recomendações para trabalhos futuros incluem: i) estudos que não se restrinjam à coleta de dados baseada em questionários, mas empregando outras estratégias; ii) análise de dados a partir de outras técnicas estatísticas, mais robustas; e iii) continuar estudando o contexto da aplicação destas práticas em outros setores da indústria, para obter um panorama mais amplo sobre a gestão de custos logísticos no País.

\section{Conflito de interesses}

Os autores declaram não haver conflito de interesses.

\section{Referências}

Gonçalves, T., Gaio, C. \& Silva, M. (2018). Target costing and innovationexploratory configurations: a comparison of fsQCA, multivariate regression, and variable cluster analysis. Journal of Business Research, 89, 378-384. https://doi.org/10.1016/j.jbusres.2018.01.054

Amaral, J. V. (2012). Trade-offs de custos logísticos. Dissertação (Mestrado em Contabilidadel - Faculdade de Economia, Administração e Contabilidade, Universidade de São Paulo, São Paulo.

Argueta, C. M., Cardona, O. C. S., Albán, H. M. G. \& Moreno, J. P. M. (2015). Análisis del tamaño de empaque en la cadena de valor para minimizar costos logísticos: un caso de estudio en Colombia. Estudios Gerenciales, 31(134), 111-121. https://doi.org/10.1016/j.estger.2014.06.009

Ballou, R. H. (1993). Logística Empresarial, transporte, administração de materiais, e distribuição física. São Paulo: Atlas.

Campelo, A. T. O. \& Faustino, M. K. S. (2015). Práticas de gestão de custos logísticos: um estudo de caso em uma empresa do ramo salineiro no município de Areia Branca-RN. Revista Conhecimento Contábil, 2(1), 1-14.

Castro, J. A. O., Camelo, N. S., Ospina \& Y. I. C. (2016). Costos logísticos y metodologías para el costeo en cadenas de suministro: una revisión de la literatura. Cuadernos de Contabilidad, 17(44), 377-420. http://dx.doi.org/10.11144/Javeriana.cc17-44.clmc

Congreso de Colombia. (2000). Ley 590 de 2000 Nivel Nacional. Colombia. Por la cual se dictan disposiciones para promover el desarrollo de los micros, pequeñas y medianas empresas. Recuperado em 20 de janeiro de 2017, de: http://www.alcaldiabogota.gov.co/sisjur/normas/Norma1.jsp?i=12672

Coser, T. \& Souza, M. A. de. (2017). Custo total de uso e propriedade (TCO): estudo de caso em uma indústria gráfica do Rio Grande do Sul (RS). Contabilidade Vista \& Revista, 28(1), 67-88.

Dianwei, Q. (2006, September). The Research on Logistics Cost Accounting and Management in China. En Proceedings of 2006 International Conference on Management of Logistics and Supply Chain, Sydney, Australia. Recuperado em 29 de janeiro de 2017, de: https://bit.ly/2EoVAB5

Dirección de Impuestos y Aduanas Nacionales de Colombia - DIAN. (2016). Resolução Número 000076 de 1 de dezembro de 2016. Recuperado em 5 de dezembro de 2016, de: https://bit.ly/3jaqfRe

Everaert, P., Bruggeman, W., Sarens, G., Anderson, S. R. \& Levant, Y. (2008). Cost modeling in logistics using time-driven $\mathrm{ABC}$ : experiences from a wholesaler. International Journal of Physical Distribution \& Logistics Management, 38(3), 172-191. http://dx.doi.org/10.1108/09600030810866977

Faria, A. C. \& Costa, M. F. G. (2007). Gestão de custos logísticos: custeio baseado em atividades $(A B C)$, balanced scorecard (BSC), valor econômico agregado (EVA). São Paulo: Atlas. 
Fellous, S. M. (2009). Gestão da cadeia de suprimentos no Brasil e a utilização de instrumentos da contabilidade gerencial: uma avaliação sob a perspectiva dos profissionais envolvidos. Dissertação (Mestrado em Contabilidadel - Faculdade de Economia, Administração e Contabilidade, Universidade de São Paulo, São Paulo.

Ferrin, B. G. \& Plank, R. E. (2002). Total cost of ownership models: an exploratory study. Journal of Supply Chain Management, 38(2), 18-29. https://doi.org/10.1111/j.1745-493X.2002.tb00132.x

Ferreira, F. B. \& Schnorr, C. (2011). Práticas de gestão de custos logísticos: um estudo de caso em empresa gaúcha do setor alimentício. In Anais do Congresso Brasileiro de Custos. Recuperado 6 de fevereiro de 2016, de: https://anaiscbc.emnuvens.com.br/anais/article/view/621

González, C. C. A. (2014). Sistema para la gestión logística empresarial. Sotavento MBA, (23), 32-41. Recuperado em 22 de março de 2016, de: https://papers.ssrn.com/sol3/papers.cfm?abstract_id=2539230

González C., J. A. (2015). Contratación logística en Colombia: implementación de un operador logístico integral. Semestre Económico, 18(38), 215-237.

Guerreiro, R., Rodrigues B. S. \& Vazquez V. M. (2008). Cost-to-serve measurement and customer profitability analysis. The International Journal of Logistics Management, 19(3), 389-407. http://dx.doi.org/10.1108/09574090810919215

Hälinen, H. M. (2015). Understanding the concept of logistics cost in manufacturing. Recuperado em 5 de maio de 2016, de: https://bit.ly/2QmYusw

Ibargüen, C. E. C. \& Cortés, J. A. Z. (2012). Análisis de costos variables logísticos para Pymes. Escenarios: empresa y territorio, 1, 191-205.

Islam, D. M. Z., Meier, J. F., Aditjandra, P. T., Zunder, T. H. \& Pace, G. (2013). Logistics and supply chain management. Research in Transportation Economics, 41(1), 3-16. http://dx.doi.org/10.1016/j.retrec.2012.10.006

Jaimes, W. A., Díaz, C. F. \& Serna, M. D. A. (2011). Modelos logísticos para la optimización del transporte de racimos de fruto fresco de palma de aceite en Colombia. Ciencia e Ingeniería Neogranadina, 21(1), 89-113.

Kang, M. (2015). Activity-based Costing Research on Enterprise Logistics Cost Management. Business and Management Research, 4(2), 18-21. https://doi.org/10.5430/bmr.v4n2p18

Krajnc, J., Logožar, K. \& Korošec, B. (2012). Activity-based management of logistic costs in a manufacturing company: a case of increased visibility of logistics costs in a Slovenian paper manufacturing company. PrometTraffic Transportation, 24(1), 15-24.

LaLonde, B. J. \& Pohlen, T. L. (1996). Issues in supply chain costing. The International Journal of Logistics Management, 7(1), 1-12. https://doi.org/10.1108/09574099610805395

Márquez, F. P. G., Pardo, I. P. G. \& Nieto, M. R. M. (2015). Competitiveness based on logistic management: a real case study. Annals of Operations Research, 233(1), 157-169. https://doi.org/10.1007/s10479-013-1508-z

Mejía-Argueta, C., Higuita-Salazar, C. \& Hidalgo-Carvajal, D. (2015). Metodología para la oferta de servicio diferenciado por medio del análisis de costo de servir. Estudios Gerenciales, 31(137), 441-454. http://dx.doi.org/10.1016/j.estger.2015.08.002

Namazi, M. (2016). Time-driven activity based costing: theory, applications and limitations. Iranian Journal of Management Studies, 9(3), 457-482. http://doi.org/10.22059/IJMS.2016.57481

Nigam, S. (2010). Breaking the Barriers: women in logistics. Available at SSRN. http://dx.doi.org/10.2139/ssrn.2428088

Nge, D., Rattanawiboonsom, V., Mahmood, A. \& Rurkwararuk, W. (2016). The critical factors affecting logistics activities: an empirical study of garment industry in Myanmar. International Business Management, 10(14), 2597-2607.

Öker, F. \& Adigüzel, H. (2016). Time-driven activity based costing: an implementation in a manufacturing company. Journal of Corporate Accounting \& Finance, 22(1), 75-92. https://doi.org/10.1002/jcaf.20646

Pohlen, T. L. \& LaLonde, B. J. (1994). Implementing activity-based costing $(A B C)$ in logistics. Journal of Business Logistics, 15(2), 1-23.

Santos, T. F. D., Gonçalves, A. T. P. \& Leite, M. S. A. (2016). Logistics cost management: insights on tools and operations. International Journal of Logistics Systems and Management, 23(2), 171-188. https://doi.org/10.1504/IJLSM.2014.065500

Servera-Francés, D. (2010). Concepto y evolución de la función logística. Innovar, 20(38), 217-234.

Silva, T. F., Gonçalves, A. T. \& Leite, M. S. (2014). Logistics cost management: insights on tools and operations. International Journal of Logistics Systems and Management, 19(3), 329-346. https://doi.org/10.1504/IJLSM.2014.065500
Souza, M. A., Zwirtes, A., Rodniski, C. M. \& Borghetti, J. C. (2013a). Gestão de Custos Logísticos: um estudo das práticas utilizadas por uma cooperativa agroindustrial catarinense. Contexto, 13(23), 7-22.

Souza, A. A., Schnorr, C. \& Ferreira, F. B. (2013b). Práticas de gestão de custos logísticos: estudo de caso de uma empresa do setor alimentício. Revista Contemporânea de Contabilidade, 10(19), 3-32. https://doi.org/10.5007/2175-8069.2013v10n19p3

Souza, M. A., Rempel, C. \& Silva, J. L. R. (2014). Práticas de gestão de custos logísticos: estudo de caso em uma empresa do setor de bebidas. Revista de Contabilidade e Organizações, 8(21), 25-35. https://doi.org/10.11606/rco.v8i21.61266

Souza, M. A., Weber, E. L. \& Campos, R. H. (2015). Práticas de gestão de custos logísticos internos: estudo de caso em empresa moveleira do sul do Brasil. Revista Contemporânea de Contabilidade, 12(25), 27-46. https://doi.org/10.5007/2175-8069.2015v12n25p27

Song, H. \& Wang, L. (2009). The status and development of logistics cost management: evidence from Mainland China. Benchmarking: An International Journal, 16(5), 657-670. http://dx.doi.org/10.1108/14635770910987869

Škerlič, S. \& Muha, R. (2016). The importance of systems for controlling logistics costs in the supply chain: a case study from the Slovenian automotive industry. Promet-Traffic Transportation, 28(3), 299-310. https://doi.org/10.7307/ptt.v28i3.1881

Škrinjar, J. P., Ivaković, Č. \& Šimunović, L. (2008). Activities and education in logistics. Promet-Traffic \& Transportation, 20(2), 125-132.

Slusarczyk, В. (2014). Logistics costs measurement at enterprises. Економічний часопис-XXI, (11-12), 97-100.

Stapleton, D., Pati, S., Beach, E. \& Julmanichoti, P. (2004). Activity-based costing for logistics and marketing. Business Process Management Journal, 10(5), 584-597. http://dx.doi.org/10.1108/14637150410559243

Uyar, M. (2014). A research on total cost of ownership and firm profitability. The International Institute for Science, Technology and Education (IISTE), 5(1), 9-16.

Vargas, S. B., Coser, T. \& Souza, M. A. (2016). Mensuração dos custos logísticos: estudo de caso em uma indústria gráfica. Contabilidade Vista \& Revista, 27(1), 63-87.

Wong, Y.-Y., Maher, T. E., Nicholson, J. D. \& Gurney, N. P. (2000). Strategic alliances in logistics outsourcing. Asia Pacific Journal of Logistics and Marketing, 12(4), 3-21.

Xiao, S. L., Sun, Y. \& Shi, S. Q. (2009). Study on modern logistics cost management. In 16 th International Conference on Industrial Engineering and Engineering Management, Beijing, China. http://dx.doi.org/10.1109/ICIEEM.2009.5344537

Yang, Y. M. (2011). The willing for companies to build the logistics cost control system of supply chain and its influence factors. Advanced Materials Research, 228-229, 162-168. https://doi.org/10.4028/www.scientific.net/AMR.228-229.162 